\title{
Online Tracking Control of Autonomous Mobile Robot Utilizing Optimal Formulation*
}

\author{
Nobuto HIRAKOSO**, Takahiro TAKIZAWA**, Masaaki ISHIHARA** \\ and Kouzou AOKI** \\ ${ }^{* *}$ Depatment of Mechanical Engineering, Gunma National College of Technology \\ 580 Toriba-machi Maebashi, Gunma Pref., Japan \\ E-mail: hirakoso@mech.gunma-ct.ac.jp
}

\begin{abstract}
In this study, the objective is to build a wheeled mobile robot which can move independently avoiding obstacles. To move autonomously, this robot is enabled to detect obstacles' shapes and conduct self-localization. Also, this robot can move by tracking trajectories designed by the robot itself, based on the information about the obstacles' shapes and the robot's position and attitude angle. The optimal trajectories which lead the robot to its destination are designed by using a unique optimization method. As convergent calculation is performed by setting the variables within a certain range in this proposed optimization method, the optimal solutions can be obtained approximately, even in cases where there is a difference between the number of input and output variables, and when the nonlinearity is strong with restraint conditions. In this thesis, the effectiveness of the optimal track designing method used is proven and the method deemed as appropriate.
\end{abstract}

Key words: Autonomous Robot, Optimize Control, Track Design

\section{Introduction}

Nowadays, the robot technology is progressing at a remarkable pace and has been adapted into a variety of fields. In near future robotics would be playing a more vital role in contributing towards a better human life. Robots are now used in large numbers of fields such as agriculture, forestry, medical, welfare ${ }^{(1)}$, disaster prevention, and rescuing in disaster scenes ${ }^{(2)}$. Along with the diversification of advanced robotics, the functions of a robot became the focal point among engineers.

In this study, a wheeled mobile robot which is capable of running autonomously by perceiving surrounding environments and avoiding obstacles independently is developed. A robot as such is exceptionally useful for occasions where signals from relay and sending stations are unavailable. It is also expected to be able to navigate its way independently through complex paths and rubbles especially in places where it is judged to be too hazardous to the human body ${ }^{(3)(4)}$. In this thesis, in order to successfully realize the autonomous wheeled movement, the following methods are described. A range sensor ${ }^{(5)(6)}$ is used for measuring the shapes and forms of obstacles, the Hough transformation used for making functions out of shapes ${ }^{(7)(8)}$, robot localization ${ }^{(9)}$, and the optimal track design involving convergent calculations for online control algorithms ${ }^{(10)}$. As arithmetic processing improves over the years, it is now possible to carry out image processing's calculations and optimization problem ${ }^{(11)}$ with converging calculations, by utilizing the calculation ability

Received 1 Dec., 2011 (No. 11-0752) [DOI: 10.1299/jsdd.6.310]

Copyright $\odot 2012$ by JSME 
developed for onboard computers. Here, the authors contrive an optimal track design by adapting performance index in the online control system. While conventional optimal control methods are adapted only for linear equations with restraint conditions and enable robots to conduct only discrete segment movement ${ }^{(12)}$,present optimal control method is adapted for not only linear and nonlinear equations, but also cases with difference in the number of input and output parameters. In this study, the authors focus on implementing optimized calculation in the online control system, and propose the optimized calculation algorithm with little calculation load. The proposed optimized control method can be applied to wide constraint conditions used for formulation of the performance index.

To verify the effectiveness for the proposed control system, an autonomous moving experiment using the wheeled mobile robot was carried out. In this thesis, the effectiveness of the autonomous mobile robot is shown, and the obtained results documented.

In this paper, formulation of the motion's performance of the built wheeled mobile robot by using the feedforward control system is discussed in Chapter 2. In Chapter 3, the methods for detection and recognition of obstacles and wall surfaces, which are keys for a successful autonomous running, are introduced. In Chapter 4, the formulation of the optimal solutions by numerical solution with convergent calculation method for online control system is introduced. And methods for track-designing which the robot moves on are discussed. And finally in Chapter 5, a simple experiment is carried out and the results obtained are reported and evaluated.

\section{Wheeled Mobile Robot}

To construct an autonomous movement system, a wheeled mobile robot with two wheels was first produced. In this chapter, the abstract and the motion property of the produced robot are mentioned.

\subsection{Abstract of the wheeled mobile robot}

Components used in this wheeled mobile robot are listed in Table 1. And the appearance of the wheeled mobile robot is shown in Fig. 1. The wheeled mobile robot has a wheel on each side of the body, and it performs translatory motion and rotating motion by controlling angular velocities of each wheel independently. The motion of the body is defined by utilizing a velocity control system composed of a servo system, which the control system uses brushless motors and motor drivers as an actuator system that rotates the wheels. The center of the body of the robot is equipped with a laser range sensor which detects the shapes of obstacles.

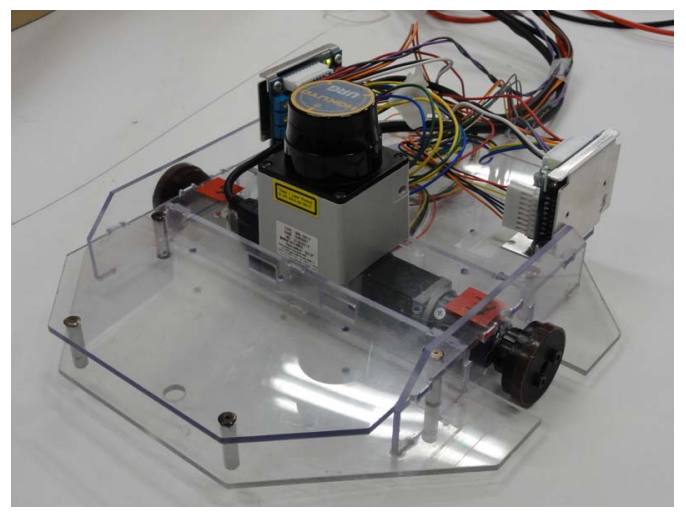

Fig. 1 Photo of Wheeled Mobile Robot 
Table1 mobile robot device

\begin{tabular}{ccc}
\hline Component & Maker Name & Product Name \\
\hline Brushless Motor & TUKASA & TG-22D-FU-64-KA \\
Motor Driver & TUKASA & TCP-S21B-22D \\
Laser range sensor & HOKUYO & URG-04LX \\
\hline
\end{tabular}

\subsection{Formulation of moving performance}

To define the motion property of the wheeled mobile robot, the property is formulated. This robot is enabled to perform translatory motion and rotating motion by providing angular velocities to its wheels on both sides. Each parameter to formulate the motion property of the body is shown in Fig. 2. Tracks of the robot can be defined by the difference between revolution velocities of both wheels, and all the tracks are described as circular arcs. Note that the translatory motion can be categorized as rotating motion because a straight line is a circular arc which radius is infinite. If a rotation radius and a central angle of a circular arc are determined, the circular arc can be defined. Here, the formulation of the motion property is defined as the body angular velocity because a central angle of a circular arc is the time integration of the body angular velocity.

In this paper, as it is the main object to verify the effectiveness of the optimal track-designing method, the tracking control of the wheeled mobile robot is carried out on the flat terrain with little friction. Therefore, influences on dynamics of the robot, such as disturbance, are not considered.

The rotation radius of the body is expressed as $R[\mathrm{~m}]$ and the body angular velocity expressed as $\Omega[\mathrm{deg} / \mathrm{sec}]$. The following equations are obtained as the motion property of the body.

$$
\begin{aligned}
& R=\frac{\omega_{1}+\omega_{2}}{\omega_{1}-\omega_{2}} \cdot \frac{l}{2} \\
& \Omega=\frac{r}{l}\left(\omega_{1}-\omega_{2}\right)
\end{aligned}
$$

Here, angular velocities of each wheel are $\omega_{1}[\mathrm{deg} / \mathrm{sec}]$ and $\omega_{2}[\mathrm{deg} / \mathrm{sec}]$, the radius of the wheel $r[\mathrm{~m}]$, and the distance between the wheels $l[\mathrm{~m}]$.

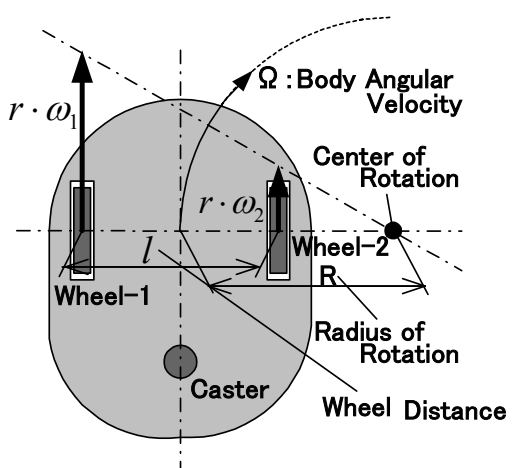

Fig. 2 Motion Formulation for Wheeled Movement Robot

\subsection{Inverse problem formulation of the motion property}

To control the track of the wheeled mobile robot practically, it is necessary to formulate the inverse problem where the angular velocities of wheels are set as command values. When the rotation radius and the angular velocity of the body are known, the following equations are obtained as angular velocity of each wheel. Here, the characters and physical quantities used are the same as those in $\S 2.2$. 


$$
\begin{aligned}
& \omega_{1}=\frac{\Omega}{r}\left(R+\frac{l}{2}\right) \\
& \omega_{2}=\frac{\Omega}{r}\left(R-\frac{l}{2}\right)
\end{aligned}
$$

\section{Detection of obstacle shapes}

\subsection{Laser range sensor}

It is necessary to construct a system which is able to detect shapes of obstacles so that the wheeled mobile robot can move independently, avoiding obstacles ${ }^{(13)(14)(15)(16)}$. Here the distance and orientation between the body and the obstacles are measured by the laser range sensor. To conduct an experiment on detecting obstacles' shapes by using the laser range sensor, an obstacle shown in Fig. 3 is used, and the data is obtained by the laser range sensor equipped on the center of the body. The result (image data), which is measured by the laser range sensor, is shown in Fig. 4. The laser range sensor used this time has a wide measuring range of $240 \mathrm{deg}$.

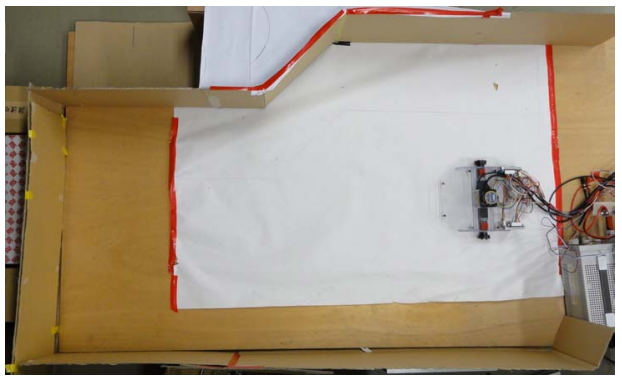

Fig. 3 Shape of obstacle

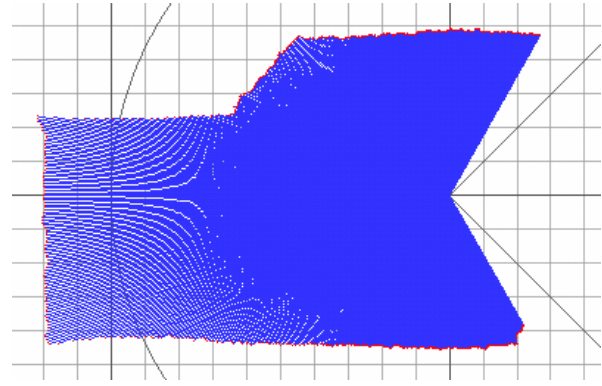

Fig. 4 Result of URG output data

\subsection{Method for recognizing obstacle shapes}

Shapes of obstacles are outputted as discrete points in the polar coordinate system by the laser range sensor, which contains information about orientation and distance. As these data are outputted as discrete points, it is necessary to derive functions from these data by some sort of method, in order to distinguish the points as obstacles' shapes. Since the major objective is to put obstacles avoidance and autonomous movement into practice in this study, a simple curve fitting suffices for defining functions of obstacles' shapes, and the functions are defined as gatherings of line segments. Therefore, the Hough transform ${ }^{(7)(8)}$, which can recognize obstacles' shapes as gatherings of line segments, is used as a method for defining functions.

The major procedures of the Hough transform are as follows.

1. The shapes of obstacles are obtained as discrete data by the laser range sensor.

2. The obtained terrain data are transformed into images.

3. The shapes of the obstacle are shown as gatherings of line segments by the Hough transform.

4. The characteristic points are fixed at the points where two line segments intersect, and coordinates $\left(x_{c n}, y_{c n}\right)$ and inclinations $\left(a_{c n}, b_{c n}\right)$ are assigned to the points.

Note that the inclinations $\left(a_{c n}, b_{c n}\right)$ here indicate the constant terms of the equation $a_{n} x+b_{n} y=0$ of line segments as shown in Fig. 5 . 


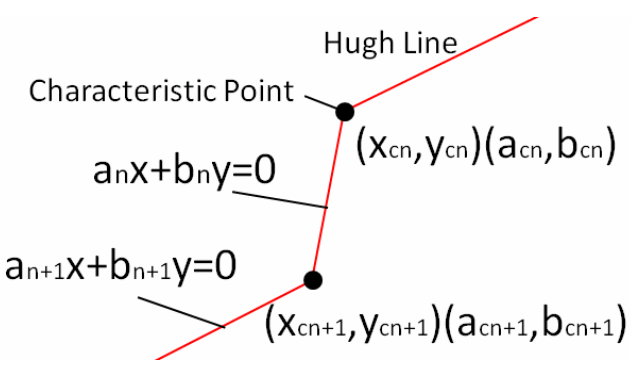

Fig. 5 Characteristic Point

\subsection{Experiment on detecting obstacle shapes}

To verify the effectiveness of using the Hough transform method, an experiment on curve fitting of line segments was conducted. The Hough transform was conducted by using the discrete data obtained, introduced in Fig. 4. The result of the Hough transform for the data is shown in Fig. 4 where they are defined as functions consisting gatherings of line segments. In Fig. 6, the faint distribution points represent discrete data obtained by the laser range sensor, while the marks represent endpoints defining line segments. Full lines represent the line segments of which curve fittings are done.

This result shows that the Hough transform method is effective for defining functions for line segments.

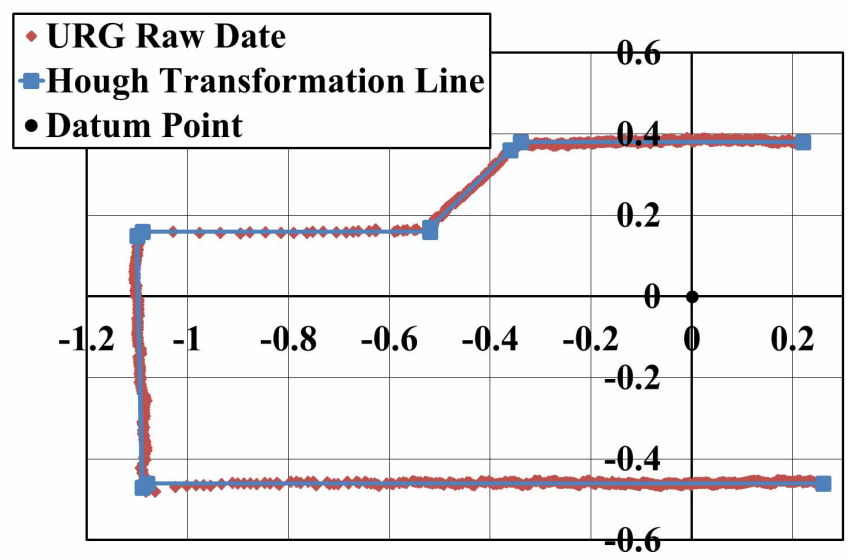

Fig. 6 Result of obstacle shape reorganization

\section{Algorithm of autonomous movement}

\subsection{Procedure for autonomous movement}

In order to have the wheeled mobile robot move autonomously, a track-designing method which uses the function data of the obstacle's shape is mentioned. Under online control, it is necessary to construct a control system, while considering the burdens of arithmetic processing. In this study, a track-designing which uses the optimization method is proposed. Procedures for autonomous movement are as follows.

1. Detection of obstacles' shapes by the laser range sensor and curve fitting of line segments by using the Hough transform are conducted.

2. Present position ${ }^{(17)}$ and attitude angle of the robot are specified depending on the relative changes of the functions of obstacles' shapes obtained in Procedure 1.

3. Target point, to where the body should move and its attitude angle are defined.

4. The movement track is designed by combining two circular arcs and connecting it to the current position and the point obtained in Procedure 3 
5. The wheels are then moved with angular velocities obtained with Eqs. (3) and (4), based on the circular track obtained in Procedure 4.

Regarding the procedures mentioned above, detailed explanations for each process are shown hereafter.

\subsection{Localization for the wheeled mobile robot}

To conduct track designing, it is also necessary to know the absolute position of the body. In this study, it is assumed that obstacles are static, and a method for localization ${ }^{(8)}$ is treated. This method estimates the coordinate and the attitude angle of the body autonomously by defining relative movements of the obstacles as results of movements of the robot itself. It is also treated that the coordinate and the attitude angle of the body estimated from the displacement of the characteristic point nearest to the body. Here, let the characteristic point be $\left(x_{c n}, y_{c n}\right)\left(a_{c n}, b_{c n}\right)$ and the moved characteristic point $\left(x_{c n}^{\prime}, y_{c n}^{\prime}\right)\left(a_{c n}^{\prime}, b_{c n}^{\prime}\right)$. Next, the travel of the body is expressed by the following equations.

$$
\begin{aligned}
& d \theta=\sin ^{-1}\left(\frac{a_{c n} b_{c n}^{\prime}-b_{c n} a_{c n}^{\prime}}{a_{c n}^{2}+b_{c n}^{2}}\right) \\
& \left(\begin{array}{l}
d x \\
d y
\end{array}\right)=\left(\begin{array}{l}
x_{c n}^{\prime} \\
y_{c n}^{\prime}
\end{array}\right)-\left(\begin{array}{cc}
\cos d \theta & -\sin d \theta \\
\sin d \theta & \cos d \theta
\end{array}\right)\left(\begin{array}{l}
x_{c n} \\
y_{c n}
\end{array}\right)
\end{aligned}
$$

Therefore, the present position of the body can be obtained by

$$
\left(\begin{array}{l}
x_{n} \\
y_{n} \\
\theta_{n}
\end{array}\right)=\left(\begin{array}{l}
x_{n-1} \\
y_{n-1} \\
\theta_{n-1}
\end{array}\right)-\left(\begin{array}{ccc}
\cos d \theta & -\sin d \theta & 0 \\
\sin d \theta & \cos d \theta & 0 \\
0 & 0 & 1
\end{array}\right)\left(\begin{array}{l}
d x \\
d y \\
d \theta
\end{array}\right)
$$

where $\left(x_{n-1}, y_{n-1}, \theta_{n-1}\right)$ is the past position of the body, $\left(x_{n}, y_{n}, \theta_{n}\right)$ represents the current position and the second term on the right hand side of the equation stands for absolute displacement. And the sampling period of localization is set to $0.5 \mathrm{~s}$ in consideration of the calculation load on the arithmetic processing unit.

\subsection{Method for setting a target point}

As it is considered to be an experimental condition that the wheeled mobile robot moves at a constant distance from the obstacle, the distance is set to about $0.20 \mathrm{~m}$ this time. Therefore, when the wheeled mobile robot moves, target points are set by the following way so that the robot can fulfill the condition roughly. Two line segments with the nearest characteristic point $\left(x_{c n}, y_{c n}\right)\left(a_{c n}, b_{c n}\right)$ as intersection are both shifted by $0.20 \mathrm{~m}$ along the normal direction. By doing this, a new intersection point appears. This new point is then set as the target point $\left(x_{n+1}, y_{n+1}, \theta_{n+1}\right)$. The relation between the characteristic point and the target point is shown in Fig. 7 where $\theta_{n+1}$ is obtained as follows.

$$
\theta_{n+1}=\tan ^{-1}\left(-\frac{a_{c n}}{b_{c n}}\right)
$$




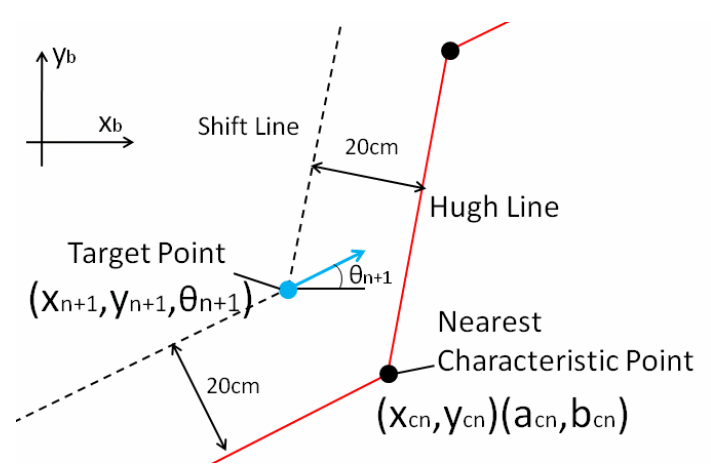

Fig. 7 Setting a Target Point

\subsection{Optimal track design}

In this section, the way to design an optimal track is discussed. In this study, optimal track design proposed by the authors is used for obtaining the solutions with minimizing the performance index formatting constraint condition constructed from unknown variables. As it is impossible to solve these unknown variables analytically, numerical solutions are obtained by changing the unknown variables within a certain finite range so that the performance index is minimized. The merits of this optimal method are as follows:

1. Application field of this optimal method for formulation of constraint condition so wide.

2. Even if the constraint condition is constructed from equations with strong nonlinearity, the numerical solutions are easily obtained.

3. If the number of unknown variables is different from the number of the equations for the constraint condition, proposed optimal problem can be solved.

Next, the track design using present optimal method is discussed. First, an arc is drawn based on the target point's coordinate and attitude angle. At the same time, another arc is drawn based on the current coordinate and attitude angle of the robot. The two arcs are then combined to create the track of the robot. The boundary conditions of the target and the current points are shown in Fig. 8. As outlined earlier in this thesis, the motion of the wheeled mobile robot can be defined by circular arcs, and its track can be specified by using only two circular arcs. A formulation example is shown hereafter. Here, the coordinate and the attitude angle of the target point are expressed as $\left(x_{n+1}, y_{n+1}, \theta_{n+1}\right)$ while the coordinate and attitude angle of the current point are expressed as $\left(x_{n}, y_{n}, \theta_{n}\right)$. These six variables are taken as known quantities, and the desired radius of two circular arcs and their respective central angles are $\left(r_{1}, \varphi_{1}\right),\left(r_{2}, \varphi_{2}\right)$ as four unknown variables. This leads to solving a problem with four unknown quantities $\left(r_{1}, \varphi_{1}\right),\left(r_{2}, \varphi_{2}\right)$ using the six known variables previously mentioned. However, as a lot of errors accumulate in the information of the current position $\left(x_{n}, y_{n}, \theta_{n}\right)$, the authors assume that $\left(x_{n}, y_{n}, \theta_{n}\right)=(0,0,0)$ and the authors calculate to obtain only the relative increment between $\left(x_{n+1}, y_{n+1}, \theta_{n+1}\right)$ and $\left(x_{n}, y_{n}, \theta_{n}\right)$. By doing this, the number of known quantities becomes three from six, with four unknown quantities. Nonetheless, solving this problem analytically still remains an impossible task.

$$
\begin{aligned}
& R_{1}=\left(r_{1}+r_{2}\right) \cdot \sin \left(\varphi_{1}\right)-r_{2} \cdot \sin \left(\theta_{n+1}\right)-x_{n+1} \\
& R_{2}=r_{1}-\left(r_{1}+r_{2}\right) \cdot \cos \left(\varphi_{1}\right)+r_{2} \cdot \cos \left(\theta_{n+1}\right)-y_{n+1} \\
& R_{3}=r_{1} \cdot \varphi_{1}+r_{2} \cdot\left(\varphi_{1}-\theta_{n+1}\right)-\sqrt{x_{n+1}^{2}+y_{n+1}^{2}}
\end{aligned}
$$


where $R_{1}, R_{2}, R_{3}$ are residuals. $R_{1}$ is the difference between the target $\mathrm{x}$ coordinate value and the $\mathrm{x}$ coordinate value changed in accordance with Table 2 for four unknown variables $\left(r_{1}, \varphi_{1}\right),\left(r_{2}, \varphi_{2}\right) . R_{2}$ is the difference between the target $\mathrm{y}$ coordinate value and the $\mathrm{y}$ coordinate value changed in accordance with Table 2 for four unknown variables $\left(r_{1}, \varphi_{1}\right),\left(r_{2}, \varphi_{2}\right)$. R3 is the equation to minimize path length. Let weight coefficients be $k_{1}, k_{2}, k_{3}$, and the performance index $J$ is shown by the following equation.

$$
J=\int\left(k_{1} \cdot R_{1}{ }^{2}+k_{2} \cdot R_{2}{ }^{2}+k_{3} \cdot R_{3}{ }^{2}\right) d t
$$

Values $k_{1}=0.4, k_{2}=0.4, k_{3}=0.2$ are placed respectively. Here, with the minimum value of performance index $J$, the unknown quantities of $\left(r_{1}, \varphi_{1}\right),\left(r_{2}, \varphi_{2}\right)$ are sought. The range of $\left(r_{1}, \varphi_{1}\right),\left(r_{2}, \varphi_{2}\right)$ are shown in Table 2 . Each weight coefficient can be arbitrarily set by designers to consider impact on the system. In this case, the numerical solutions are necessarily obtained.

Table 2 Variable Range for Optimal Solution

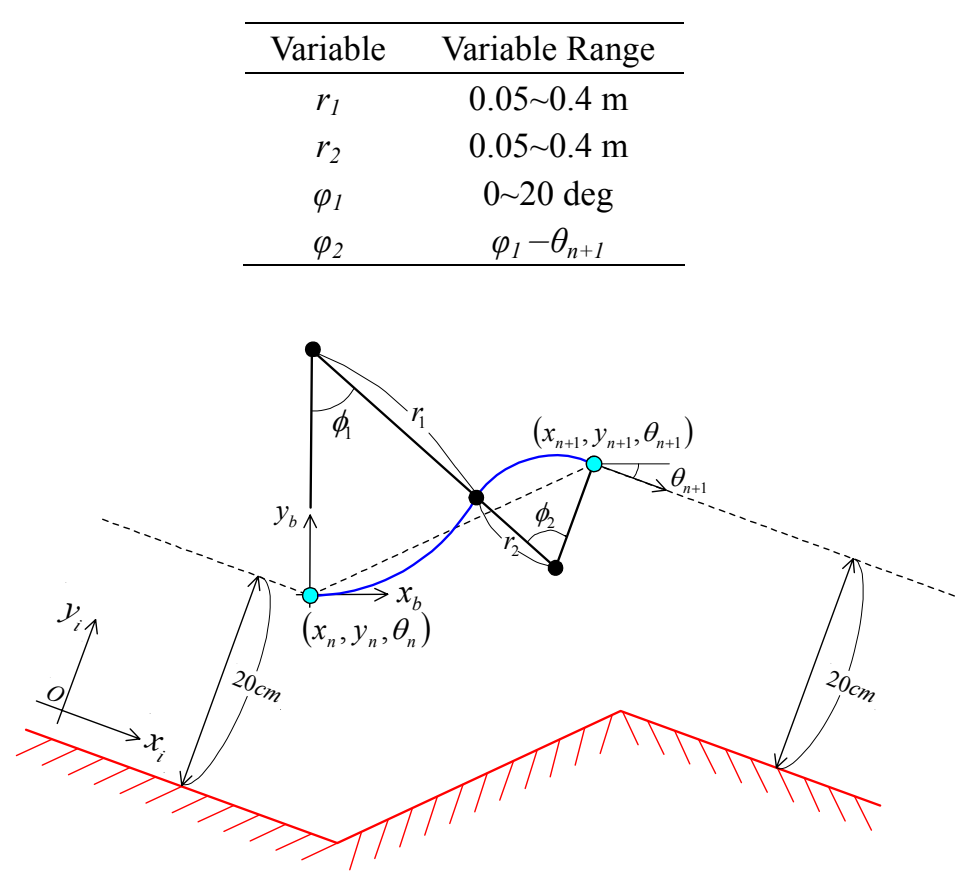

Fig. 8 Optimal Problem for Two Point Boundary Condition

\subsection{Tracking control}

As the next process of autonomous movement, the wheels are moved by angular velocities based on the circular track obtained in $\S 4.4$ in accordance with Eqs. (3) and (4). Equations. (3) and (4) are equations defined analytically. Because this wheeled mobile robot is still in a trial stage, the robot's body is not equipped with its arithmetic processing unit and power source. This means that the power supply and communication with the body are conducted with cable. Also, a notebook computer is used as the arithmetic processing unit, and control of the robot is carried out through the medium of an AD/DA PC card. The specifications of the notebook computer and the AD/DA PC card used in this study are shown in Table. 3.

The servo system of both wheels inputs angular velocity commands calculated by the arithmetic processing unit into the motor drivers as analog signals, and the motor drivers in turn drives the brushless motors. 
Table 3 Experimental System Component

\begin{tabular}{ccc}
\hline Component & Maker Name & Product Name \\
\hline AD/DA PC Card & CONTEC & ADA16-8/2(CB)L \\
Note PC & LENOVO & 2714-A21 \\
\hline
\end{tabular}

\section{Experiment on avoiding obstacles under autonomous running}

To verify the effectiveness of the automatic function of the wheeled mobile robot using the optimization method, an experiment on avoiding obstacles under autonomous running is conducted and its results reported as follows.

\subsection{Experimental condition}

The shape of the obstacle used as the experimental condition for the autonomous running test is shown in Fig. 9. It is crucial that the obstacle can evaluate the property of the autonomous running. Therefore, the same obstacle was used in the experiment on autonomous running shown in Fig. 3, 4 and 6, introduced in Chapter 3. This shape of the obstacle is designed to test whether the robot can autonomously move, avoiding the bending portion of the obstacle without a collision, with the right side in Fig. 9 as a starting point.

Arithmetic processing and the power supply to the robot are conducted under cabled communication. The arithmetic processing unit controls the robot through the medium of the $\mathrm{AD} / \mathrm{DA}$ board. In this experiment, the velocity of the body can be set arbitrarily. However, in order to reduce the influence of slipping between the wheels and the ground, the velocity of the body is set to $0.05 \mathrm{~m} / \mathrm{s}$ in consideration of the calculation period for localization.

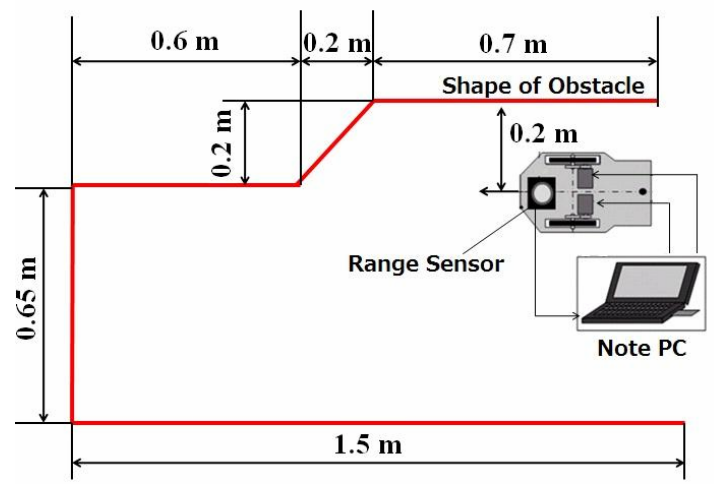

Fig. 9 Experimental Condition for Autonomous Running Test

\subsection{Result of the experiment on avoiding an obstacle under autonomous running}

The result of the experiment is shown in Fig. 10(a). The running track of the wheeled mobile robot and the still image of the obstacle's shape are shown in Fig. 10(b). In Fig. 10 (a), the running track shows that the wheeled mobile robot moved, avoiding the obstacle perfectly. Also, it indicates that the robot moved successfully at a rough constant distance from the obstacle.

The errors in the laser range sensor's measuring, curve fitting for the obstacle's shape, target point setting, trajectory tracking are listed as error factors. Regarding the wheel's driving system, the accuracy of tracking is not satisfying this time due to slipping between the wheels and the ground besides the degradation of angular velocities of the wheels in practical running. The authors considered getting rid of these errors by taking the result of localization into the real-time feedback system. However, in the result of this experiment, the errors in localization are greater than those caused by factors like slipping between the 
wheels and the ground, and the time-lag caused by the calculation period for localization leads to gross errors when the robot moves quickly. Therefore, improvement in the accuracy of localization is not expected even if the result of localization is taken into the real-time feedback system. Moreover, when a terrain-approximation with line segments in the phase of terrain detection is carried out, a divergence of characteristic points on the obstacle occurs by approximating the terrain expressed by a single line segment with two line segments. Because target point setting and localization for the robot are conducted based on the characteristic points, it is thought that this greatly affects the errors of the autonomous running.

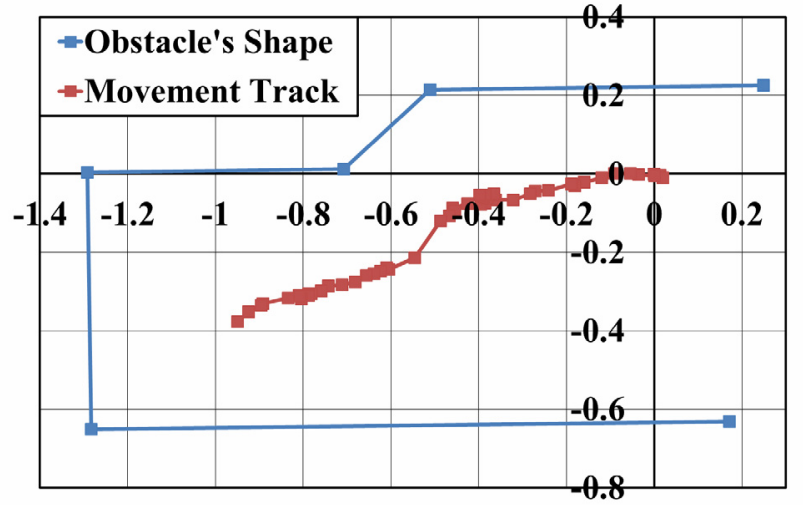

Fig. 10(a) Experimental Result for Robot Running Trajectory

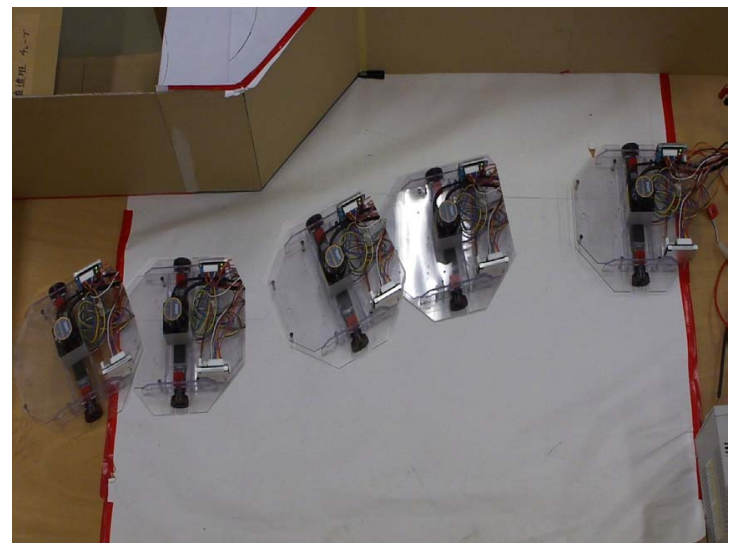

Fig. 10(b) Experimental Result for Robot Running Trajectory

\section{Summary}

In order to actualize the autonomous movement of the wheeled mobile robot which could avoid obstacles independently, the authors introduced these key methods. Firstly, the construction of the obstacles' recognition and detection system was done. Next, an optimal track-design method utilizing the boundary conditions of the current and target points was introduced into the autonomous system. To verify the effectiveness of the autonomous system composed of the mentioned methods, an experiment using a simple obstacle was carried out. The results indicates that the built robot equipped with the autonomous system successfully avoided the obstacle. In addition, the experiment also proves that the robot managed to design a track that roughly traces the shape of the obstacle and move following the track. Thus it is proven that this autonomous system, which comprises the optimal track designing method, is effective and appropriate.

As future work, fitting functions utilizing curve functions for the experiment of avoiding complicated, random obstacles' will be introduced. 


\section{References}

(1)Kaino, Y., Miwa, M. and Matsuda N., Wheel Chair Robot System For Hospital Use, JSME annual meeting, Vol. 5 (2006-09), pp.41-42.

(2)Hirakoso, N. and Kurosawa, Y., Experiment Simulated on Ground of Autonomous Flying Robot by Using Wheeled Mobile Robot - Study of Navigation Method for Constant-Level- , Proceedings of the 11th, transactions of the Society of Instrument and Control Engineers system integration (in Japanese), SI2010 (2010-12), pp.104-105.

(3)Komiyama, K., Suzuki, S., Nakazawa, D. and Nonami, K., Guidance Control for a Small-Scale Unmanned Helicopter, Transactions of motion and vibration control conference(in Japanese), Vol. 11 (2009-09), pp.181-184.

(4)Hirakoso, N., Seshimo, W. and Ishikawa, T., Experimental Study Simulated on Ground of Landform Tracing Navigation Method for Autonomous Flying Robot, Proceedings of the 53th Japan joint automatic control conference (in Japanese), No.10-253 (2010-11), pp.1041-1044.

(5)Iwakura, D., Wang, W., Nonami, K. and Haley, M., Movable Range-Finding Sensor System and Precise Automated Landing of Quad-Rotor MAV, Journal of System Design and Dynamics, Vol. 5, No. 1(2011), pp.17-42.

(6)Nakamoto, T., Yamashita, A. and Kaneko, T., 3-D MAP GENERATION BY A MOBILE ROBOT EQUIPPED WITH A LASER RANGE FINDER, Engineering laboratory report of the Institute of Electronics, Information and Communication Engineers(in Japanese), Vol. 105, No. 500(2006-01), pp.167-172.

(7)Ishidate Takashi. "Detection of straight line and circle from a picture using the Hough transformation"(in Japanese). CodeZine. (online), available from $<$ http://codezine.jp/article/ datail/153>

(8)Laarson, J., Broxvall, M and Saffiotti, A. Laser Based Corridor Detection for Reactive Navigation, Industrial Robot, Vol. 35, No.1, pp.69-79, 2008

(9)Kawai, K., Nakazawa, A., Kiyokawa, K. and Takemura, H., Path Planning using Potential Field for 3D Reconstruction of a Disaster Site by a Mobile Robot, IEICE Technical Report, Vol.105, No.612(2006-02), pp.85-90.

(10)Hirakoso, N., Ishihara, M. and Kurosawa, Y., Tracking Control of Autonomous Wheeled Mobile Robot using Optimal Track Design, Proceedings of the 12th, transactions of motion and vibration control conference(in Japanese), No. 11-6(2011-07), pp.459-463.

(11)Miyata, H., Ohki, M. and Ohkita, M., Self-Tuning of Fuzzy Reasoning by the Steepest Descent Method and Its Application to a Parallel Parking, IEICE transactions on information and systems, E79-D, No.5(1996-05), pp. 561-569.

(12) Koenig, S. and Likhachev, M., D* Lite, Proceedings of the National Conference on Artificial Intelligence(AAAI), 2002.

(13)Okawa, I. and Nonaka, K., Receding Horizon Control of Lateral Wheel Position on Obstacle Avoidance for Leg/Wheel Robots, Transactions of motion and vibration control conference(in Japanese), Vol. 11 (2009-09), pp.195-198.

(14)Fukui, Y., Nakamura, H. and Nishitani, H., Real-time Obstacle Avoidance of a Two-wheeled Mobile Robot via Minimum Projection Method, Transactions of the Society of Instrument and Control Engineers, Vol. 2 (2011-02), pp.90-99.

(15)Ohmuro, A. and Hattori, Y., Optimum Vehicle Trajectory Control for Obstacle Avoidance : A Minimax Problem of Resultant Vehicle Force(Mechanical Systems), Transactions of the Japan Society of Mechanical Engineers part C, 76(772) (2010-12), pp.3587-3594.

(16)Joochim, T. and Chamnongthai, K., Mobile Robot Navigation by Wall Following Using Polar Coordinate Image from Omnidirectional Image Sensor, IEICE transactions on information and systems, E85-D No. 1(2002-01), pp.264-274. 
(17)Wang, H., Ishimatsu, T. and Mian, J, T., Self-Localization for an Electric Wheelchair, JSME international journal. Series C, Mechanical systems, machine elements and manufacturing, Vol. 40, No. 3(1997-09), pp.433-438. 